\title{
Solid-pseudopapillary neoplasm of the pancreas in a patient with familial adenomatous polyposis: a case report
}

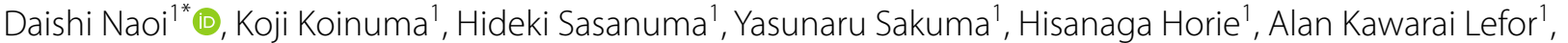 \\ Kokichi Sugano ${ }^{2}$, Mineko Ushiama ${ }^{3}$, Teruhiko Yoshida ${ }^{3}$ and Naohiro Sata ${ }^{1}$
}

\begin{abstract}
Background: Familial adenomatous polyposis (FAP) is characterized by the presence of hundreds to thousands of colonic polyps, and extracolonic manifestations are likely to occur. Pancreatic tumors are rare extracolonic manifestations in patients with FAP, among which solid-pseudopapillary neoplasm (SPN) are extremely rare. We report here a patient with an SPN of the pancreas found during the follow-up of FAP.

Case presentation: A 20-year-old woman was diagnosed with FAP 3 years previously by colonoscopy which revealed less than 100 colonic polyps within the entire colon. She complained of left upper abdominal pain and a 10-cm solid and cystic pancreatic tumor was found by computed tomography scan. Solid and cystic components within the tumor were seen on abdominal magnetic resonance imaging. Simultaneous laparoscopic resection of the distal pancreas and subtotal colectomy was performed. Histopathological findings confirmed the pancreatic tumor as an SPN without malignancy. Abnormal staining of beta-catenin was observed by immunohistochemical study. Multiple polyps in the colorectum were not malignant. Molecular biological analysis from peripheral blood samples revealed a decrease in the copy number of the promoter $1 \mathrm{~A}$ and $1 \mathrm{~B}$ region of the $A P C$ gene, which resulted in decreased expression of the APC gene.

Conclusions: A rare association of SPN with FAP is reported. The genetic background with relation to beta-catenin abnormalities is interesting to consider tumor development. So far, there are few reports of SPN in a patient with FAP. Both lesions were treated simultaneously by laparoscopic resection.
\end{abstract}

Keywords: Solid-pseudopapillary neoplasm, Familial adenomatous polyposis, Laparoscopic surgery, Case report

\section{Background}

Familial adenomatous polyposis (FAP) is an autosomal dominant disease characterized by the presence of hundreds to thousands of adenomatous polyps in the colorectum, and colon cancer is considered inevitable over a lifetime. Extracolonic manifestations are likely to occur in patients with FAP [1,2]. Pancreatic tumors are rare

\footnotetext{
*Correspondence: daishi.naoi@jichi.ac.jp

${ }^{1}$ Division of Gastroenterological, General and Transplant Surgery, Department of Surgery, Jichi Medical University School of Medicine, Yakushiji 3311-1, Shimotsuke, Tochigi 329-0498, Japan

Full list of author information is available at the end of the article
}

extracolonic manifestations in patients with FAP [3, 4], and there are few reports of solid-pseudopapillary neoplasms (SPN) in patients with FAP [5-7].

We report a patient with FAP who was diagnosed with a SPN during the follow-up, and both diseases were treated simultaneously by laparoscopic resection.

\section{Case presentation}

The patient is a 20-year-old woman diagnosed with FAP 3 years previously by colonoscopy which revealed less than 100 benign polyps involving the entire colon (Fig. 1a, b). Her father, older brother and older sister were all diagnosed with FAP and treated by 


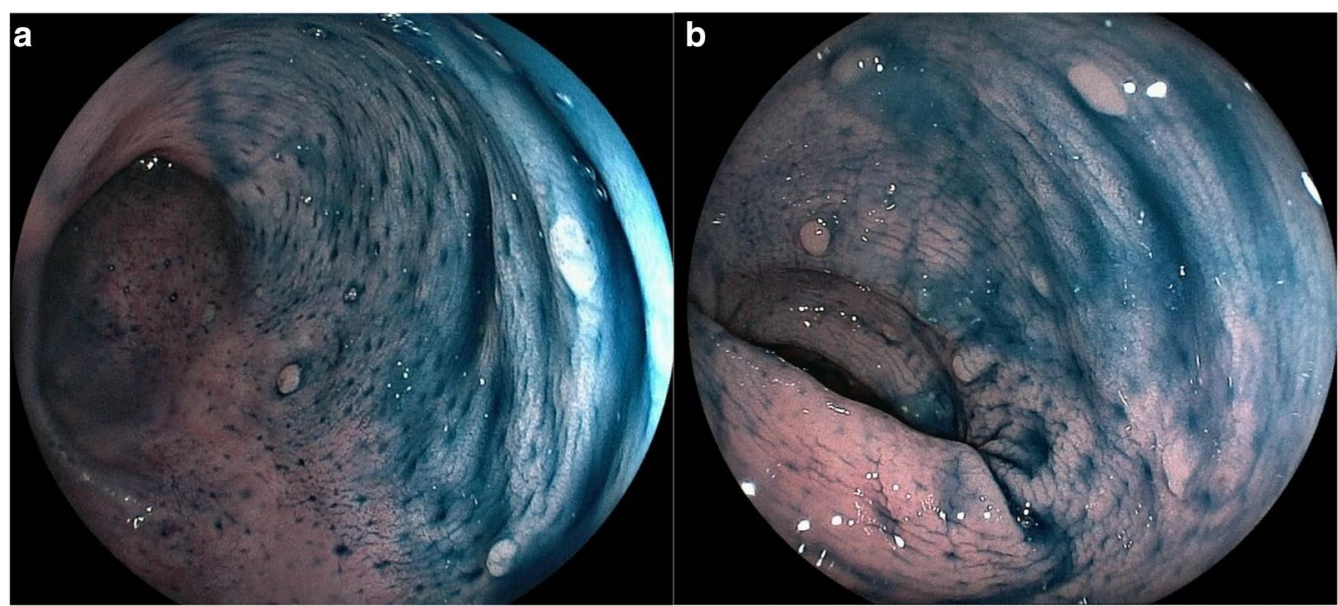

Fig. 1 Colonoscopy showed less than 100 benign polyps involving the entire colon $(\mathbf{a}, \mathbf{b})$

surgical resection. Her older brother and older sister had desmoid tumors. She presented with left upper abdominal pain and enhanced computed tomography scan showed a $10-\mathrm{cm}$ pancreatic tumor with clear margins defined by a calcified capsule. Solid and cystic components were inside the lesion (Fig. 2a, b). On magnetic resonance imaging, part of the cystic component had high intensity on T1- (Fig. 3a) and low intensity on T2-weighted images (Fig. 3b), suggesting intra-tumor bleeding. No signs of malignancy were observed. The pancreatic tumor was diagnosed as an SPN. Given that she was already 20 years old from a FAP family, prophylactic colectomy was indicated. Simultaneous laparoscopic distal pancreatectomy and subtotal colectomy with an ileo-rectal anastomosis were performed. The post-operative course was uneventful. She remains free of SPN recurrence 5 years postoperatively, although an abdominal desmoid tumor is followed up.

Macroscopically, the SPN had a gray solid component with bleeding and a multi-locular cystic component with necrosis (Fig. 4a, b). Histopathological findings of the SPN revealed loose tumor cell connectivity with cell dissociation and pseudopapillary arrangements (Fig. 5a). There was no evidence of malignancy. Immunohistochemical staining showed that both the nucleus and cytoplasm in the tumor cells stained with beta-catenin (Fig. 5b) but were negative for chromogranin A and synaptophysin. The polyps in the colorectum had no evidence of malignancy.

Protein truncation tests from an analysis of peripheral blood samples revealed no mutations in the protein coding region of the APC (adenomatous polyposis coli) gene. Analysis by the multiplex ligation-dependent
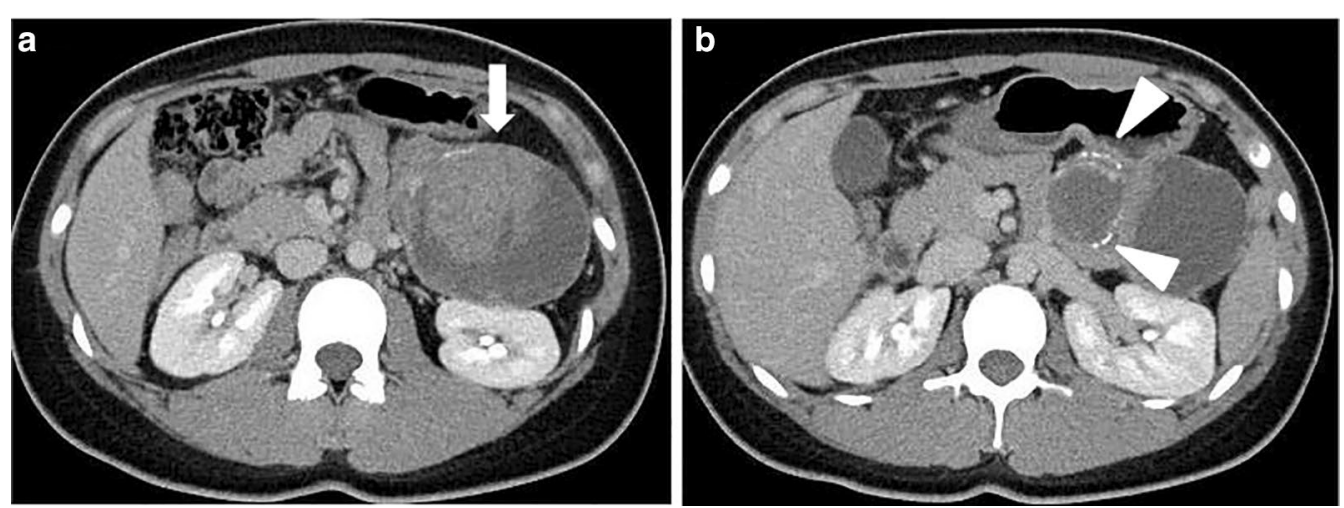

Fig. 2 Abdominal computed tomography scan images. A 10-cm clearly demarcated tumor (arrow) with solid and cystic components are seen in the pancreatic tail (a), and the capsule had calcifications (arrowhead) (b) 

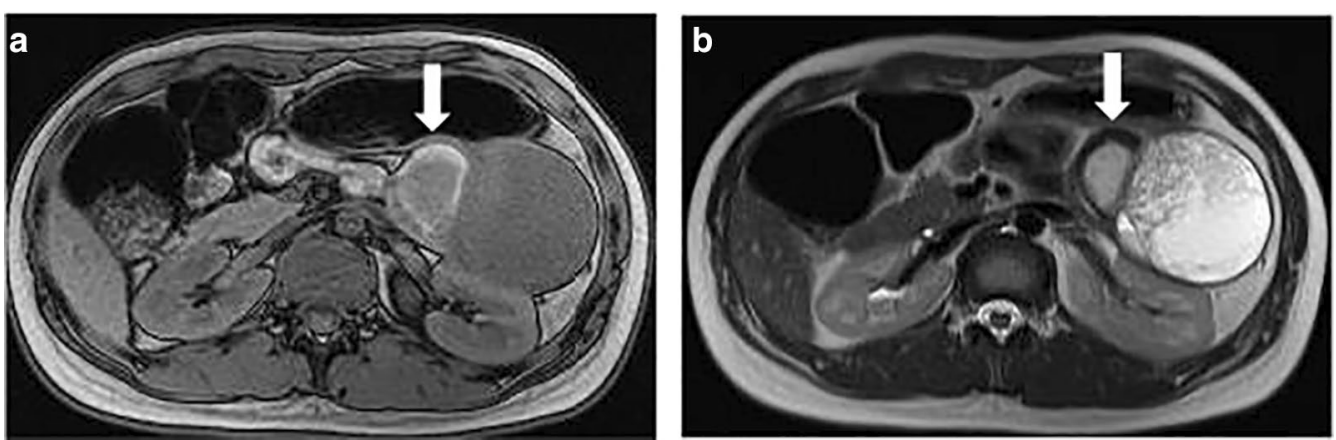

Fig. 3 Magnetic resonance imaging. The tumor had bleeding (arrow) shown by high intensity on T1-weighted images (a), and a low intensity on T2-weighted images (b)
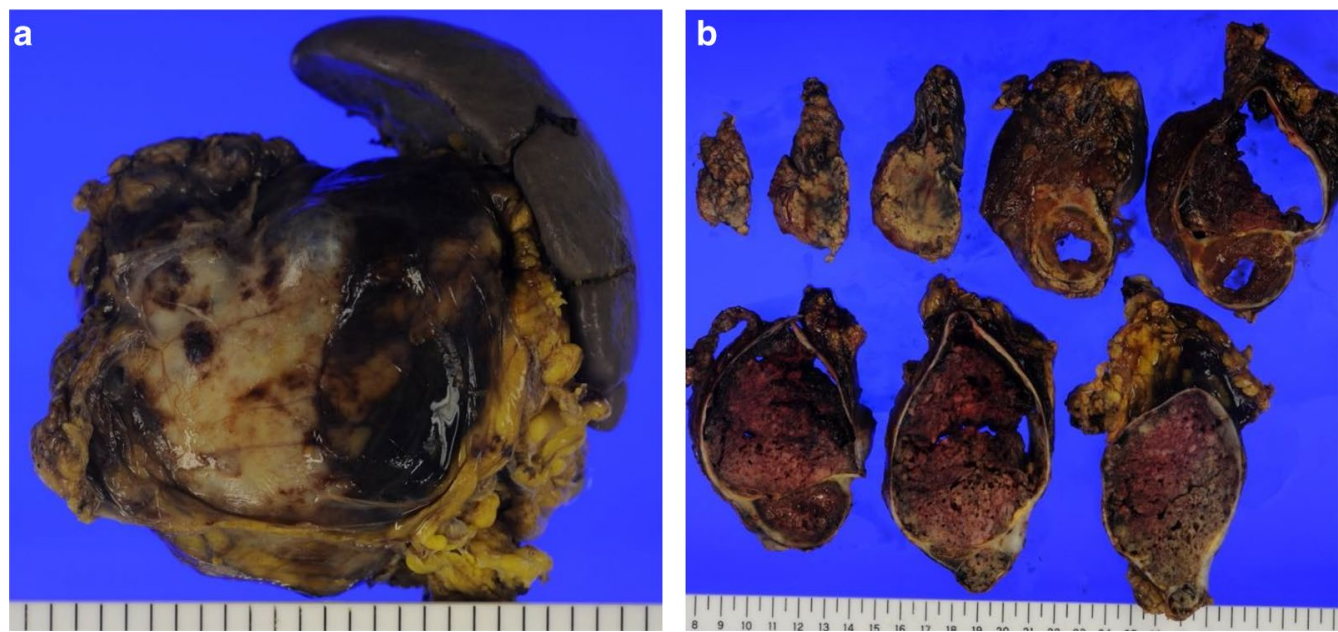

Fig. 4 Resected specimen. Gray solid component with bleeding and a multi-locular cystic component with necrosis were inside a thick capsule (a, b)
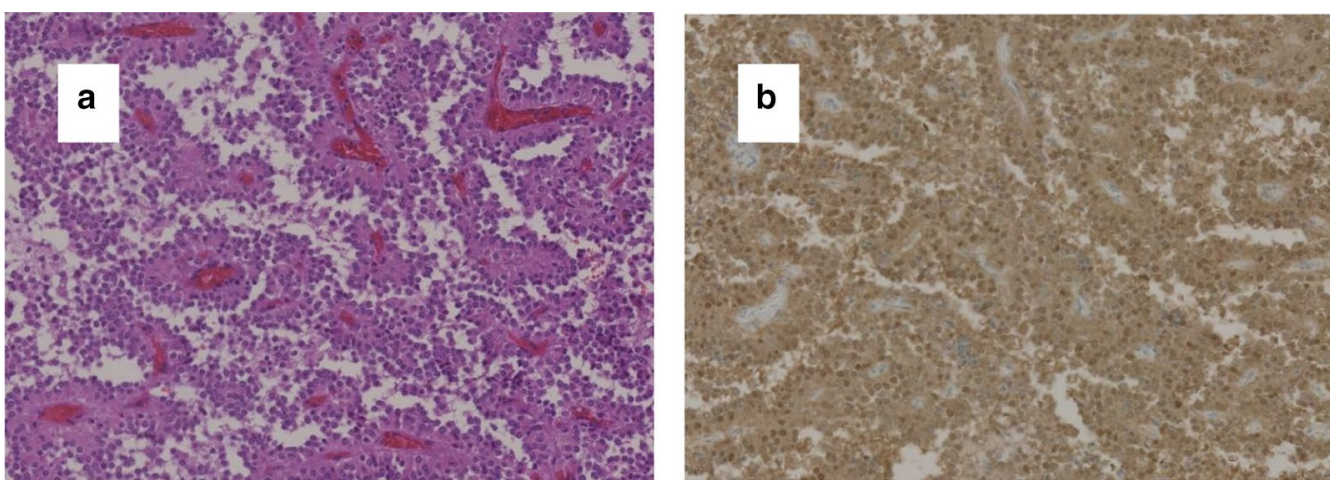

Fig. 5 Pathological findings of the pancreatic tumor (a; hematoxylin \& eosin $\times 400$ ). Immunohistochemical examination shows both nucleus and cytoplasm positive for $\beta$-catenin $(b ; \times 400)$ 


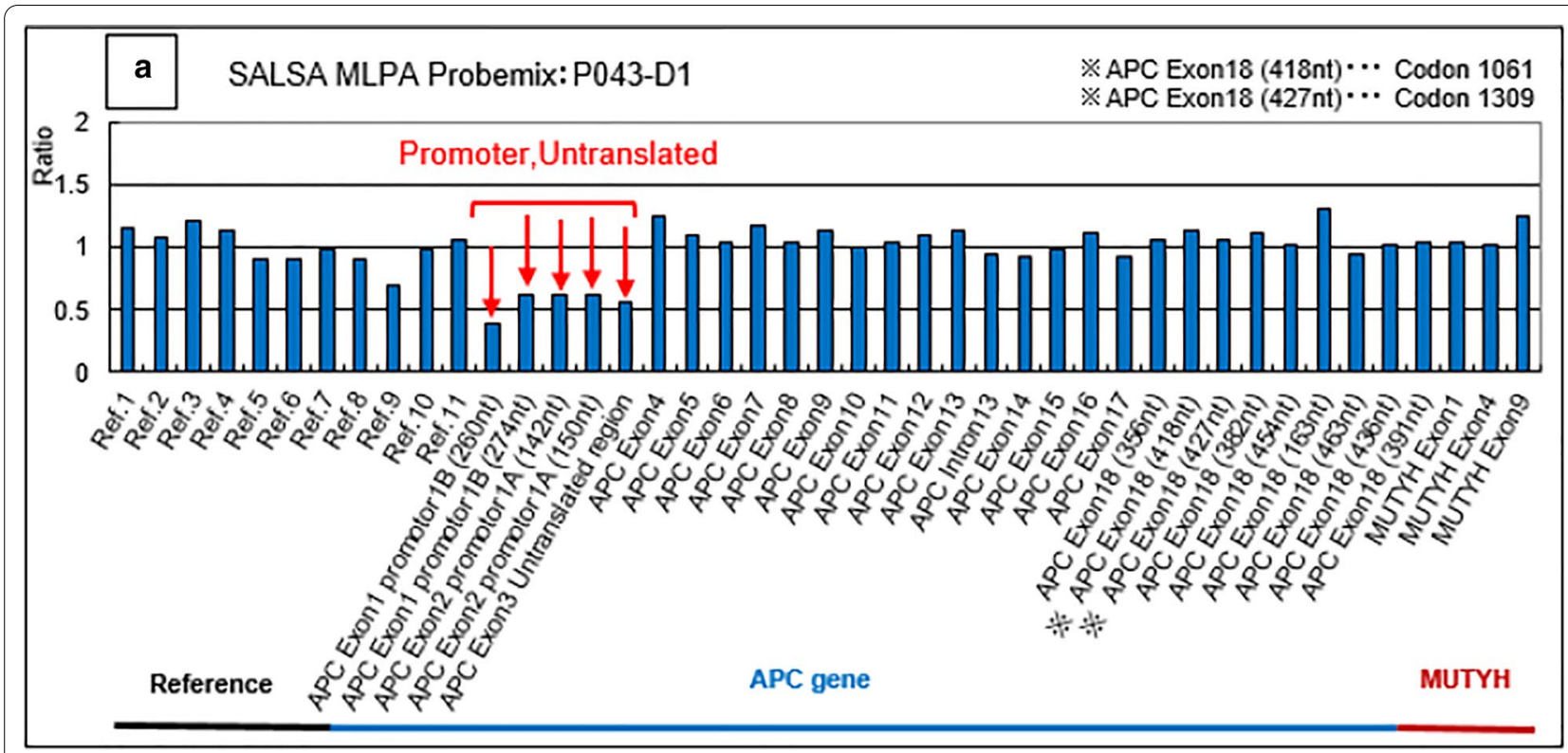

b

Genomic organization of the APC gene promoter region

chr5:112,707,509-112,738,404 / MLPA ligation site 1) -5) is designed

MLPA DEL Probe

MLPA Retained Probe

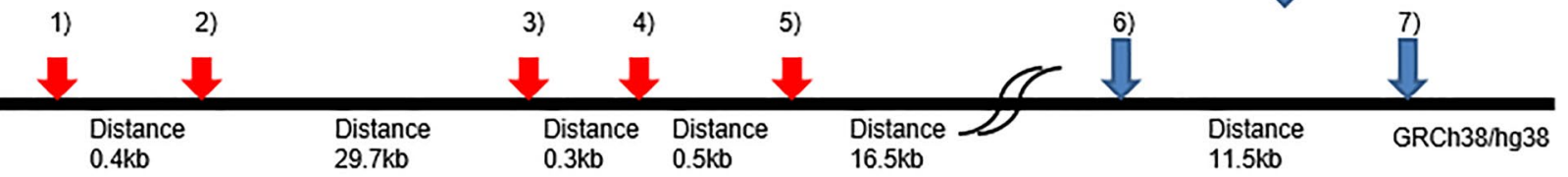

Transcript variant 1

NM_001127511.2

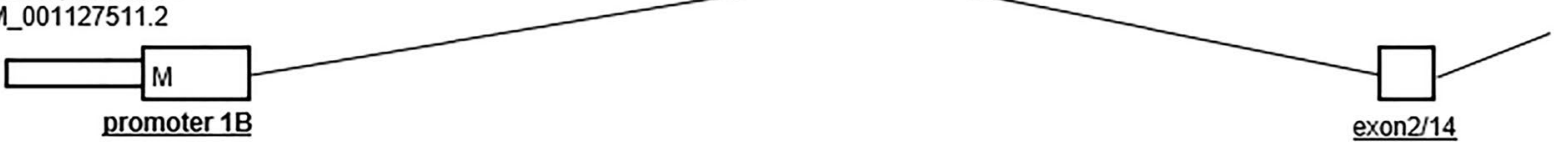

Transcript variant 2

NM_001127510.2

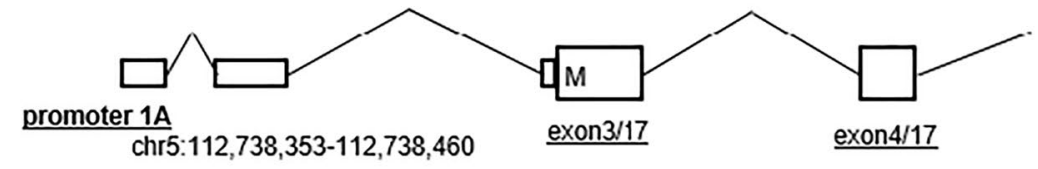

Transcript variant 3

NM_000038.5

chr5:112,738,353-112,738,460

exon $4 / 17$

chr5:112,707,498-112,707,882

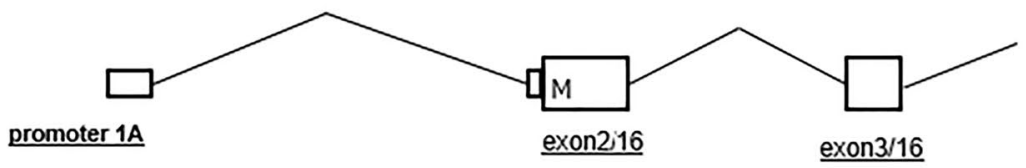

chr5:112,737,859-112,737,925

chr5:112,754,873-112,755,025

chr5:112,766,326-112,766,410

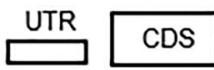

M:Met:ATG

Fig. 6 Analysis by the multiplex ligation-dependent probe amplification technique. A decrease in the copy number of the promoter 1A and 1B region of the APC gene was revealed (a). Genomic organization of the promoter region of the APC genes and arrows indicate the positions of probes hybridizing the APC gene (b) 
probe amplification (MLPA) technique that can quantitatively analyze exonic deletions and duplication revealed a decrease in the copy number of the promoter $1 \mathrm{~A}$ and $1 \mathrm{~B}$ region of the $A P C$ gene (Fig. $6 \mathrm{a}, \mathrm{b}$ ), which resulted in decreased expression of the $A P C$ gene in this patient [8]. Genetic analysis of the resected specimen was not performed.

\section{Discussion}

FAP is caused by mutations of the $A P C$ gene. Loss of function mutation in the $A P C$ gene is associated with an accumulation of intracellular beta-catenin protein, which leads to loss of control of normal beta-catenin signaling, which promotes colorectal adenoma formation. If prophylactic colectomy is not performed, colon cancer is considered inevitable over a lifetime.

Extracolonic manifestations are likely to occur in patients with FAP [1,2], including benign (osteomas, epidermoid cysts, desmoid tumor, congenital hypertrophy of the retinal pigment epithelium, fundic gland polyposis) and malignant (adenocarcinoma of the duodenum, thyroid, pancreas, biliary tract, stomach, and tumor of the liver or central nervous system) lesions $[1-3,9,10]$. Pancreatic tumors are rare extracolonic manifestations in patients with FAP, most of which were reported to be ductal adenocarcinoma of the pancreas. Although, the life-time risk of pancreatic carcinoma is estimated at about $2 \%$ in patients with FAP, the risk has been estimated to be more than four times that of the general population [3, 4]. However, there are few reports of SPN in patients with FAP [5-7]. SPN is a rare tumor with low malignant potential, representing about 0.17 to $2.7 \%$ of pancreatic tumors [11]. Most are seen in young females and are in the body or tail of the pancreas. Surgical resection is recommended for treatment, as $95 \%$ or more can be cured by complete resection [12-14]. Genetically, beta-catenin mutations frequently occur in patients with SPN and has been thought to play a critical role in the tumorigenesis of SPN. An abnormal nuclear accumulation of beta-catenin protein is observed in almost $100 \%$ of SPN lesions $[15,16]$. It is interesting that betacatenin abnormalities are observed in both SPN and FAP, although their association in tumorigenesis is still unclear.

In previously reported patients with FAP who develop SPN, the SPN were diagnosed during follow-up of colon cancer in two patients $[5,6]$. The other was a 14-year-old girl who had a history of SPN resection and was subsequently diagnosed with FAP [7]. In the present patient, SPN was diagnosed during the follow-up of FAP. Our institution has sufficient experience performing laparoscopic colectomy and laparoscopic distal pancreatectomy, so we decided to perform laparoscopic resection of both lesions. Precise preoperative diagnoses made it possible to perform a minimally invasive surgery.

This patient had been followed as attenuated FAP (AFAP) due to the small number of colonic polyps seen on colonoscopy. AFAP is characterized by the presence of less than 100 colonic polyps, more proximally located, and a later age at diagnosis of colon cancer compared to classic FAP. AFAP is estimated to represent about 8-15\% of all FAP, and its clinical phenotype is milder with a lower incidence of extracolonic manifestations [1, 17]. Siblings of this patient developed desmoid tumors, which also developed in this patient. MLPA analysis elucidated a decrease in the copy number of the promoter $1 \mathrm{~A}$ and $1 \mathrm{~B}$ region of the $A P C$ gene, which was different from previous reports in which the mutations in the $A P C$ gene associated with AFAP have mainly been presented in the $5^{\prime}$ upstream exons, in exon 9 and in the distal $3^{\prime}$ end [15, 18]. Accordingly, this patient is likely to be classical FAP despite the small number of colonic polyps. It is difficult to assess whether a patient has AFAP at a younger age, careful follow-up including the occurrence of extracolonic manifestations is required.

\section{Conclusion}

We report a patient with SPN and FAP. The genetic background of the lesions with relation to beta-catenin abnormalities is interesting to consider tumor development and coexistence. There have been few previous case reports of SPN in a patient with FAP, and both diseases were treated simultaneously by laparoscopy.

\section{Abbreviations \\ FAP: Familial adenomatous polyposis; SPN: Solid-pseudopapillary neoplasm; APC: Adenomatous polyposis coli; MLPA: Multiplex ligation-dependent probe} amplification; AFAP: Attenuated familial adenomatous polyposis.

\section{Acknowledgements}

Not applicable.

\section{Authors' contributions}

$\mathrm{DN}, \mathrm{KK}, \mathrm{HS}, \mathrm{YS}, \mathrm{HH}$, NS were engaged in the patient's care including the surgery and prepared the manuscript. KS, MU, TY were involved in the genetic counseling and DNA analysis and interpretation of the APC gene for this patient. KK and AL helped in drafting the manuscript and interpretation of data. All authors have read and approved the final.

Funding

All authors have no funding regarding this paper.

\section{Availability of data and materials Not applicable.}

\section{Ethics approval and consent to participate}

The need for ethical approval for this paper was waived by the committee of Jichi Medical University Hospital. Genetic Counseling and subsequent DNA testing of the APC gene was performed at the division of the genetic counseling clinic, Tochigi Cancer Center and the department of genetic medicine and services, National Cancer Center Hospital, Tokyo, according to 
the protocol approved by the IRB at the Tochigi Cancer Center (Study Nos. A-019 and A-291).

\section{Consent for publication}

Written informed consent was obtained from the patient for publication of this case report and accompanying images.

\section{Competing interests}

All authors declare no conflicts of interest regarding the publication of this paper.

\section{Author details}

${ }^{1}$ Division of Gastroenterological, General and Transplant Surgery, Department of Surgery, Jichi Medical University School of Medicine, Yakushiji 3311-1, Shimotsuke, Tochigi 329-0498, Japan. ${ }^{2}$ Genome Center, Genetic Counseling Clinic, Tochigi Cancer Center Research Institute, Tochigi, Japan. ${ }^{3}$ Department of Genetic Medicine and Services, National Cancer Center Hospital, Tokyo, Japan.

Received: 14 January 2021 Accepted: 21 January 2021

Published online: 28 January 2021

\section{References}

1. Vasen HF, Moslein G, Alonso A, Aretz S, Bernstein I, Bertario L, et al. Guidelines for the clinical management of familial adenomatous polyposis (FAP). Gut. 2008;57:704-13.

2. Maire F, Hammel P, Terris B, Olschwang S, O'Toole D, Sauvanet A, et al. Intraductal papillary and mucinous pancreatic tumour: a new extracoIonic tumour in familial adenomatous polyposis. Gut. 2002:51:446-9.

3. Groen EJ, Roos A, Muntinghe FL, Enting RH, de Vries J, Kleibeuker JH, et al. Extra-intestinal manifestations of familial adenomatous polyposis. Ann Surg Oncol. 2008;15:2439-50.

4. Burt RW. Colon cancer screening. Gastroenterology. 2000;119:837-53.

5. Inoue T, Nishi Y, Okumura F, Mizushima T, Nishie H, Iwasaki H, et al. Solid pseudopapillary neoplasm of the pancreas associated with familial adenomatous polyposis. Intern Med. 2015;54:1349-55.

6. Ruo L, Coit DG, Brennan MF, Guillem JG. Long-term follow-up of patients with familial adenomatous polyposis undergoing pancreaticoduodenal surgery. J Gastrointest Surg. 2002;6:671-5.
7. Farahmand F, Shoaran M, Fariborzi M, Ashjaei B, Monajemzadeh M, Mehdizadeh M. Pancreatic pseudopapillary tumor in association with colonic polyposis. J Med Med Sci. 2012;3:447-51.

8. Yamaguchi K, Nagayama S, Shimizu E, Komura M, Yamaguchi R, Shibuya T, et al. Reduced expression of APC-1B but not APC-1A by the deletion of promoter $1 B$ is responsible for familial adenomatous polyposis. Sci Rep. 2016;6:26011.

9. Dinarvand P, Davaro EP, Doan JV, Ising ME, Evans NR, Phillips NJ, et al. familial adenomatous polyposis syndrome: an update and review of extraintestinal manifestations. Arch Pathol Lab Med. 2019;143:1382-98.

10. Iwama T, Tamura K, Morita T, Hirai T, Hasegawa H, Koizumi K, et al. A clinical overview of familial adenomatous polyposis derived from the database of the Polyposis Registry of Japan. Int J Clin Oncol. 2004;9:308-16.

11. Papavramidis T, Papavramidis S. Solid pseudopapillary tumors of the pancreas: review of 718 patients reported in English literature. J Am Coll Surg. 2005;200:965-72.

12. Romics L Jr, Olah A, Belagyi T, Hajdu N, Gyurus P, Ruszinko V. Solid pseudopapillary neoplasm of the pancreas - proposed algorithms for diagnosis and surgical treatment. Langenbecks Arch Surg. 2010;395:747-55.

13. Peng $\mathrm{CH}$, Chen DF, Zhou GW, Yang WP, Tao ZY, Lei RQ, et al. The solidpseudopapillary tumor of pancreas: the clinical characteristics and surgical treatment. J Surg Res. 2006;131:276-82

14. Hosokawa I, Shimizu H, Ohtsuka M, Kato A, Yoshitomi H, Furukawa $\mathrm{K}$, et al. Preoperative diagnosis and surgical management for solid pseudopapillary neoplasm of the pancreas. J Hepatobiliary Pancreat Sci. 2014;21:573-8.

15. Chetty R, Salahshor S, Bapat B, Berk T, Croitoru M, Gallinger S. Intraductal papillary mucinous neoplasm of the pancreas in a patient with attenuated familial adenomatous polyposis. J Clin Pathol. 2005:58:97-101.

16. Wood LD, Hruban RH. Pathology and molecular genetics of pancreatic neoplasms. Cancer J. 2012;18:492-501.

17. Roncucci L, Pedroni M, Mariani F. Attenuated adenomatous polyposis of the large bowel: present and future. World J Gastroenterol. 2017;23:4135-9.

18. Knudsen AL, Bisgaard ML, Bülow S. Attenuated familial adenomatous polyposis (AFAP). A review of the literature. Fam Cancer. 2003;2:43-55.

\section{Publisher's Note}

Springer Nature remains neutral with regard to jurisdictional claims in published maps and institutional affiliations.

\section{Submit your manuscript to a SpringerOpen ${ }^{\circ}$ journal and benefit from:}

- Convenient online submission

- Rigorous peer review

- Open access: articles freely available online

- High visibility within the field

- Retaining the copyright to your article

Submit your next manuscript at $\boldsymbol{\nabla}$ springeropen.com 